\title{
Animal models of Parkinson's disease and their applications
}

This article was published in the following Dove Press journal:

Journal of Parkinsonism and Restless Legs Syndrome

12 July 2016

Number of times this article has been viewed

\section{Hyun Jin Park \\ Ting Ting Zhao \\ Myung Koo Lee}

Department of Pharmacy, Research Center for Bioresource and Health, College of Pharmacy, Chungbuk National University, Cheongju, Republic of Korea
Correspondence: Myung Koo Lee Department of Pharmacy, Research Center for Bioresource and Health, College of Pharmacy, Chungbuk National University, I, Chungdae-ro, Seowon-gu, Cheongju 28644, Republic of Korea Tel +82432612822

Fax +82 432762754

Email myklee@chungbuk.ac.kr
Abstract: Parkinson's disease (PD) is a progressive neurodegenerative disorder that occurs mainly due to the degeneration of dopaminergic neuronal cells in the substantia nigra. L-3,4Dihydroxyphenylalanine (L-DOPA) is the most effective known therapy for PD. However, chronic L-DOPA administration results in a loss of drug efficacy and irreversible adverse effects, including L-DOPA-induced dyskinesia, affective disorders, and cognitive function disorders. To study the motor and non-motor symptomatic dysfunctions in $\mathrm{PD}$, neurotoxin and genetic animal models of PD have been widely applied. However, these animal models do not exhibit all of the pathophysiological symptoms of PD. Regardless, neurotoxin rat and mouse models of PD have been commonly used in the development of bioactive components from natural herbal medicines. Here, the main animal models of PD and their applications have been introduced in order to aid the development of therapeutic and adjuvant agents.

Keywords: Parkinson's disease, neurotoxin animal models, genetic animal models, adjuvant therapeutics

\section{Introduction}

Parkinson's disease (PD) is a progressive neurological disorder that occurs mainly due to the degeneration of dopaminergic neuronal cells in the substantia nigra pars compacta (SNpc). ${ }^{1}$ L-3,4-Dihydroxyphenylalanine (L-DOPA) is the most effective known therapy for treating the characteristic motor symptoms of PD including slowness, rigidity, resting tremor, and postural instability. ${ }^{2,3}$ Other drugs including dopamine agonists and anticholinergics are also used to control the symptoms of the movement disorders in PD. ${ }^{4}$ However, chronic L-DOPA administration results in a loss of drug efficacy and irreversible adverse effects. Moreover, it leads to the development of severe motor fluctuations such as L-DOPA-induced dyskinesia. ${ }^{5}$ The most common type of dyskinesia can occur in association with high concentrations of L-DOPA in the brain and maximum improvement in the motor responses during L-DOPA administration. ${ }^{6}$ In addition, patients with PD suffer from a variety of non-motor disorders that appear at the early or late stages of PD, including affective disorders, such as anxiety disorders and depression, and cognitive function disorders, such as learning and memory impairments. ${ }^{7,8}$ Although L-DOPA therapy has been found to improve anxiety disorders in $\mathrm{PD},{ }^{9}$ in general, the non-motor disorders are not improved or are even sometimes worsened by chronic L-DOPA administration. ${ }^{10,11}$

Recently, the etiology, pathology, and molecular mechanisms of PD have been revealed by using animal and cellular models. ${ }^{12-15}$ Furthermore, by using animal models, 
many bioactive and natural adjunctive agents have been developed that relieve the symptoms of PD (described in the "Experimental applications of animal models and the related clinical studies" section). Here, the main animal models of PD and their applications are introduced in order to aid the development of therapeutic and/or adjuvant agents.

\section{Neurotoxin models 6-Hydroxydopamine}

The classic animal model of PD is the 6-hydroxydopamine (6-OHDA) model. The neurotoxic effects of 6-OHDA are due to oxidative stress that is triggered by the formation of reactive oxygen species (ROS) after entering the neuron via the dopamine transporter. ${ }^{16,17}$ Stereotaxic injection of 6-OHDA into the SNpc or the striatum as a unilateral or bilateral model induces neuronal cell death of the tyrosine hydroxylase (TH)-containing neurons in each rat and mouse brain, which decreases the dopamine levels in the TH-positive terminals of the striatum. ${ }^{18}$ Although 6-OHDA does not induce the formation of Lewy body-like inclusions like those observed in $\mathrm{PD}, 6-\mathrm{OHDA}$ reportedly interacts with $\alpha$-synuclein ${ }^{19}$ that is the main component of Lewy bodies. ${ }^{20}$ In addition, examining the turning behavior of the animal in response to amphetamine or apomorphine after the unilateral application of 6-OHDA indicates the extent of the induced SNpc or striatal lesion, ${ }^{21,22}$ and this method has also been used to test the efficacy of potential PD therapeutics.

Moreover, 6-OHDA has been found in the human caudate nucleus and in the urine of L-DOPA-treated patients with $\mathrm{PD},{ }^{23,24}$ suggesting that 6-OHDA may play a role in the pathogenesis of PD as an endogenous hydroxylated metabolite of dopamine. However, 6-OHDA-lesioned animal model including the other neurotoxin models is different from the insidious progression of PD and does not completely reproduce the clinical symptom and pathology of PD. ${ }^{25}$

\section{I-Methyl-4-phenyl-I,2,3,6- tetrahydropyridine}

The compound 1-methyl-4-phenyl-1,2,3,6-tetrahydropyridine (MPTP) induces neurotoxicity in the nigrostriatal dopaminergic neurons in the brain of mice, rats, cats, dogs, monkeys, and other higher mammals. After a student was injected with an analog of the synthetic opioid meperidine, he showed severe PD-like bradykinesia that improved with L-DOPA. ${ }^{26}$ And then, the MPTP, which causes the PD-like symptoms, was identified from the synthetic meperidine analog as a by-product (review). ${ }^{26}$ MPTP is metabolized to the active metabolite 1-methyl-4-phenylpyridinium (MPP+) by monoamine oxidase type $\mathrm{B}^{27} \mathrm{MPP}^{+}$is taken up into the neuron by the dopamine transporter, where it then blocks the complex I site of the mitochondrial electron chain, ${ }^{28}$ thus initiating other intercellular reactions and multisystemic lesions, including oxidative stress, energy failure, and inflammation.

The species of nonhuman primate PD model are the rhesus macaque, common marmoset, squirrel monkey, African green monkey, and cynomolgus, and the baboon (review). ${ }^{26,29,30}$ The PD models of nonhuman primates have behavioral, neuroanatomical and age-related impairments correspondent with the symptoms of PD patients. ${ }^{31,32}$ Chronic MPTP-induced monkey models of PD also show dopaminergic cell loss, $\alpha$-synuclein aggregation, $\alpha$-synuclein upregulation, and neuritic $\alpha$-synuclein pathology. ${ }^{29,30,33}$ Comparisons to neuropathological data show that the MPTP-induced dopaminergic neuronal cell death in monkeys is also identical to that observed in PD. ${ }^{34}$ However, the neurodegenerative process in PD is thought to be progressive over a course of years in the human intoxicated with MPTP, although the active phase of neurodegeneration shows within a few days of the MPTP injection. ${ }^{26,35}$

\section{Rotenone}

Rotenone is a lipophilic herbicide and an insecticide from Leguminosa plants that crosses the blood-brain barrier and impairs oxidative phosphorylation in the mitochondria of rodents. ${ }^{36}$ Rotenone models show various hallmarks of PD, including complex I blockade, behavioral dysfunctions, inflammation, synuclein aggregation, Lewy body-like formations, and oxidative stress. ${ }^{37}$ However, because of the difficulties associated with using rotenone to generate a model of PD, limited data have been reported on the dopamine depletion in the nigrostriatal system of this model. ${ }^{38}$

\section{Paraquat}

Paraquat (1,1'-dimethyl-4,4'-bipyridinium) is used widely as an herbicide in agriculture, and it exhibits a structural resemblance to $\mathrm{MPP}^{+}{ }^{39}$ However, unlike $\mathrm{MPP}^{+}$, paraquat exerts deleterious effects on dopaminergic neurons through oxidative stress-mediated damage of lipids, proteins, DNA, and RNA by generating ROS, superoxide radicals, hydrogen peroxide, and hydroxyl radicals in mice. ${ }^{40}$ The systemic application of paraquat to mice reduces their motor activity and induces a dose-dependent loss of striatal TH-positive neurons. ${ }^{41,42}$ In addition, paraquat induces an increase in $\alpha$-synuclein and the formation of Lewy-like bodies in dopaminergic SNpc 
neurons. ${ }^{10,43}$ However, in several cases of paraquat-induced PD models, there is not a patent decrease in the striatal dopaminergic innervation, and there is only a transient decrease in the striatal $\mathrm{TH}$ levels, ${ }^{42,43}$ suggesting that it takes care to make the paraquat model.

\section{Methamphetamine and other derivatives}

Methamphetamine has neurotoxic effects on the nervous system that cause not only functional deficits but also structural alterations. ${ }^{10}$ However, although selective dopaminergic or serotonergic neuronal cell loss occurs in rodents following the administration of high doses of methamphetamine, this model is not very reliable; ${ }^{44}$ the results only produce a long-term loss of TH enzyme but are not examined in the PD-dependent behavioral tests.

Tetrahydropapaveroline and salsolinol, which are found in the urine of patients with PD following chronic L-DOPA administration, have been shown to be neurotoxic, as they form ROS in the dopaminergic neurons. ${ }^{45-47}$

In addition, organic metals including manganese and carbon disulfide $\left(\mathrm{CS}_{2}\right)$ cause the neurodegeneration of subthalamic neuronal cells and neurobehavioral problems. ${ }^{48}$ However, it is suggested that these metals can be considered specific dopaminergic toxins. ${ }^{39}$

\section{Genetic models $\alpha$-Synuclein}

$\alpha$-Synuclein is a ubiquitous and abundant 140-amino acid cytosolic protein and is the main component of the filaments that form Lewy bodies. ${ }^{20}$ The $\alpha$-synuclein gene has been identified as the cause of familial forms of PD. ${ }^{49,50}$ Specifically, three point mutations in $\alpha$-synuclein (A53T, A30P, and E46K) cause familial $\mathrm{PD},{ }^{51}$ and the expression level of $\alpha$-synuclein is a determinant of PD progression. ${ }^{52}$ Furthermore, $\alpha$-synuclein aggregation has been observed in the rotenone- and MPTPinduced animal models of PD. ${ }^{53,54}$ Knocking out $\alpha$-synuclein does not affect the development or maintenance of dopaminergic neurons. ${ }^{55}$ The $\alpha$-synuclein transgenic mouse models have been reported using human $\alpha$-synuclein gene mutation (A30P/ A53T). ${ }^{15,56}$ The $\alpha$-synuclein transgenic mouse model that expresses the wild-type gene with the regulated tetracycline system has also been reported: this model provides the loss of neurons of substantia nigra, progressive motor decrease, and cognitive impairment, but there is no fibrillary inclusion. ${ }^{57}$ Although the function of $\alpha$-synuclein was recently revealed, the continual expression of $\alpha$-synuclein is required for the disease progression, ${ }^{57}$ and the actual role of $\alpha$-synuclein in PD still remains elusive. ${ }^{10}$
In addition, $\alpha$-synuclein aggregation can be modulated by the formation of toxic oligomer by including interaction with lipids or small molecules, phosphorylation of $\alpha$-synuclein at Ser129 and Ser87, oxidative stress, and truncation (review). ${ }^{58}$ The level of total and oligomeric forms of normal and phosphorylated $\alpha$-synuclein (at Ser129) from PD patients as a prospective diagnosis marker is detected by the immune detection method of plasma samples and other Lewy body disease. ${ }^{59,60}$ Recent researches therefore suggest that the mechanism of the phosphorylation process of $\alpha$-synuclein in brain might be a sensitive and effective biomarker candidate for PD. ${ }^{13}$

\section{Leucine-rich repeat kinase 2 mutations}

Unlike $\alpha$-synuclein, leucine-rich repeat kinase 2 (LRRK2) is localized to membranes. ${ }^{61}$ Mutations in LRRK2, coding for dadarin, are the most frequent genetic cause of lateonset autosomal-dominant PD, ${ }^{52,62,63}$ and LRRK2 mutations have also appeared in sporadic PD. ${ }^{64}$ The main cause of LRRK2-related PD according to pathological observations is the presence of $\alpha$-synuclein inclusions. ${ }^{65}$ The toxicity of $L R R K 2$ mutations involves the overexpression of disease-causing mutations and is kinase- and guanosine5 -triphosphate-binding dependent. ${ }^{63,66}$ In contrast, knocking out $L R R K 2$ has no effect on dopaminergic neuronal development or maintenance. ${ }^{67}$ Moreover, LRRK2 transgenic mouse models do not show the further pathological results, which is unclear. ${ }^{15}$

\section{Parkin, phosphatase, and tensin homolog- induced novel kinase I, and DJ-I}

Currently, the autosomal-recessive causes of PD include mutations in parkin, phosphatase, and tensin homologinduced novel kinase 1 (PINK1), DJ-1, and ATP13A2. ${ }^{15,68}$ Parkin, PINK1, and DJ-1 mutations have been applied to generate animal and cellular models of PD. ${ }^{69-71}$ However, animal models that utilize ATP13A2 have not been reported. ${ }^{68}$

Parkin mutations account for $\sim 50 \%$ of the familial cases of PD and $20 \%$ of the young-onset PD cases. ${ }^{69}$ The 465 -amino acid parkin protein functions as an E3 ubiquitin ligase, which participates in the ubiquitin proteasome system. ${ }^{72}$ Mutations in parkin mostly lead to a loss of E3 ubiquitin ligase activity, which causes mitochondrial dysfunction followed by the general pathogenesis of early-onset parkinsonism. ${ }^{69}$ Constitutive knockout rodent models of these genes do not demonstrate any nigrostriatal degeneration. ${ }^{73}$ However, a recent report showed that the conditional deletion of parkin in adult mice is associated with $\mathrm{SNpc}$ neurodegeneration. ${ }^{74}$ 
PINK1 is localized to the mitochondrial intermembrane space and membrane, ${ }^{75}$ and may regulate mitochondrial calcium dynamics. ${ }^{76}$ Most of the reported mutations in PINK1 are loss-of-function mutations that affect the kinase domain. Similar to parkin knockouts, PINK1 knockout mice do not exhibit any major abnormalities; ${ }^{77}$ in particular, the number of dopaminergic neurons and the level of striatal dopamine are unchanged. In addition, parkin and PINK1 function in a common pathway and regulate mitochondrial trafficking and autopathy. ${ }^{78,79}$

$D J-1$ is a redox-sensitive molecular chaperone and regulator of antioxidants and is a member of the ThiJ/Pfpl family of molecular chaperones. ${ }^{70} \mathrm{DJ}-1$ is subcellularly localized to the cytosol, mitochondrial matrix, and intermembrane space, ${ }^{80}$ where it is ubiquitously expressed and functions in vivo as an atypical peroxiredoxin-like peroxide. ${ }^{81}$ Mutations in $D J-1$ result in reduced neuroprotective function and antioxidant activity and play a role in early-onset parkinsonism. ${ }^{82}$ Similar to parkin and PINK1 knockouts, DJ-1 knockout mice do not exhibit the major abnormal symptoms that are associated with PD. ${ }^{81}$

Finally, the ATP13A2 protein is a lysosomal membrane protein, and most mutations are retained and degraded in the endoplasmic reticulum, ${ }^{68,83}$ that leads to PD neurodegeneration and $\alpha$-synuclein accumulation. ${ }^{83,84}$

\section{Experimental applications of animal models and the related clinical studies}

Behavioral alterations in animal models of PD are related to dopaminergic neuronal activity in the nigrostriatal region. ${ }^{85}$ Chronic L-DOPA administration-induced dyskinesia is mainly related to the supersensitivity of dopaminergic neurons. ${ }^{5}$ PD-induced anxiety disorders are closely related to the dopaminergic and serotoninergic neuronal functions in the brain. ${ }^{86}$ In addition, memory dysfunctions are related to dopaminergic and $N$-methyl-D-aspartate (NMDA) neuronal functions. ${ }^{8,87,88}$

Interestingly, smoking and nicotine protect dopaminergic neurons in the MPTP mouse model, and nicotine also has protective effects in the primate MPTP model and in the 6-OHDA-, rotenone-, and paraquat-induced animal models of PD (review). ${ }^{26}$ Nicotine reduces the loss of striatal $\mathrm{TH}$ immunoreactivity induced by 6-OHDA, which leads to neuroprotection of the nigrostriatal region, but the precise mechanism remains unknown yet. ${ }^{89}$ Similarly, caffeine exerts neuroprotective effects in the MPTP, 6-OHDA, and paraquat animal models by antagonizing adenosine $\mathrm{A} 2 \mathrm{~A}$ receptors (review) ${ }^{26} \mathrm{SS} 31$, a mitochondria-targeted aromatic-cationic peptide, protects dopaminergic neurons in the MPTPinduced PD model..$^{90} \mathrm{D}$-Cycloserine, a partial agonist of the NMDA receptor, improves PD-related dementia by inducing behavioral and neurological changes in the MPTP-induced rat model of PD. ${ }^{91}$

The targets for various neuroprotective drugs and agents and the approach for promoting L-DOPA therapy in PD have been reviewed previously. ${ }^{4}$ Thus, we summarize the bioactive components and extracts that have been isolated from natural herbal medicines for the purpose of relieving and protecting against the symptoms of PD in Table 1.

\section{Effects on neurotoxin-induced behavioral dysfunction and the death of dopaminergic neurons}

In the 6-OHDA-induced rat model of $\mathrm{PD}$, the extract of Mucuna pruriens, ${ }^{92}$ ethanol extract of Gynostemma pentaphyllum, ${ }^{93}$ curcumin and naringenin, ${ }^{94}$ epigallocatechin derivatives from green tea, ${ }^{95}$ and bilobalide from Ginkgo biloba extract ${ }^{96}$ show neuroprotective effects.

In the MPTP-induced mouse and rat models of PD, G. biloba extract, ${ }^{97}$ kavain, ${ }^{98}$ ginsenoside $\operatorname{Rg} 1,{ }^{99}$ echinacoside from Cistanches salsa, ${ }^{100}$ celastrol, ${ }^{101}$ paeoniflorin from Paeoniae alba, ${ }^{102}$ resveratrol, ${ }^{103,104}$ baicalein, ${ }^{105}$ and gypenosides (GPS) ${ }^{106}$ from G. pentaphyllum exhibit protective effects. Extracts from Acanthopanax senticosus and Withania somnifera also show protective effects in the MPTP-induced rodent model of PD. ${ }^{107,108}$

In addition, purslane isolated from Portulaca oleraceae L. ${ }^{109}$ and sesamin from $A$. senticosus ${ }^{110}$ demonstrate protective effects on the rotenone-induced rat model of PD.

\section{Effects on MPTP-induced anxiety disorders}

GPS and the ethanol extract from $G$. pentaphyllum show anxiolytic effects on affective disorders by modulating the brain levels of dopamine and serotonin in the MPTP-induced mouse model of PD. ${ }^{111}$

\section{Effects on L-DOPA-induced dyskinesia}

One double-blind, placebo-controlled study showed that amantadine reduced L-DOPA-induced dyskinesia in PD. ${ }^{112}$ Pilot studies also suggest that cannabinoids and aripiprazole can reduce L-DOPA-induced dyskinesia in PD. ${ }^{113,114}$

Endocannabinoids reduce L-DOPA-induced dyskinesia in an MPTP-induced cynomolgus monkey model of PD. ${ }^{115}$ L-Stepholidine shows neuroprotective effects on L-DOPA- 
Table I The applications of PD animal models by bioactive components and herbal medicines

\begin{tabular}{|c|c|c|c|c|c|}
\hline $\begin{array}{l}\text { Bioactive } \\
\text { component/herbal } \\
\text { medicine }\end{array}$ & Toxic model & $\begin{array}{l}\text { Behavioral } \\
\text { function }\end{array}$ & Biochemical analysis & $\begin{array}{l}\text { Application for } \\
\text { the model }\end{array}$ & Reference \\
\hline Mucuna pruriens (seed) & $\begin{array}{l}\text { Intrastriatal 6-OHDA- } \\
\text { lesioned rat }\end{array}$ & $\begin{array}{l}\text { Rotational } \\
\text { behavior }\end{array}$ & None & $\begin{array}{l}\text { Test symptomatic } \\
\text { therapy }\end{array}$ & 92 \\
\hline $\begin{array}{l}\text { Gynostemma } \\
\text { pentaphyllum }\end{array}$ & $\begin{array}{l}\text { Unilateral 6-OHDA- } \\
\text { lesioned rat }\end{array}$ & $\begin{array}{l}\text { Rotational } \\
\text { behavior }\end{array}$ & $\begin{array}{l}\text { TH-immunohistochemistry } \\
\text { Dopamine levels }\end{array}$ & $\begin{array}{l}\text { Test symptomatic } \\
\text { therapy }\end{array}$ & 93 \\
\hline (ethanol extract) & $\begin{array}{l}\text { MPTP-lesioned mouse } \\
\text { (C57BL/6) }\end{array}$ & $\begin{array}{l}\text { Elevated plus-maze } \\
\text { test } \\
\text { Marble burying test }\end{array}$ & $\begin{array}{l}\text { Dopamine and serotonin } \\
\text { levels } \\
\text { TH-immunohistochemistry }\end{array}$ & $\begin{array}{l}\text { Test affective } \\
\text { disorders }\end{array}$ & 111 \\
\hline \multirow[t]{3}{*}{$\begin{array}{l}\text { Gypenosides } \\
\text { (G. pentaphyllum) }\end{array}$} & $\begin{array}{l}\text { MPTP-lesioned mouse } \\
\text { (C57BL/6) }\end{array}$ & $\begin{array}{l}\text { Pole test } \\
\text { Rotarod test }\end{array}$ & $\begin{array}{l}\text { TH-immunohistochemistry } \\
\text { Dopamine and its } \\
\text { metabolite levels } \\
\text { Glutathione levels } \\
\text { SOD activity }\end{array}$ & $\begin{array}{l}\text { Test symptomatic } \\
\text { therapy }\end{array}$ & 106 \\
\hline & $\begin{array}{l}\text { MPTP-lesioned mouse } \\
\text { (C57BL/6) }\end{array}$ & $\begin{array}{l}\text { Elevated plus-maze } \\
\text { test } \\
\text { Marble burying test }\end{array}$ & $\begin{array}{l}\text { Dopamine and serotonin } \\
\text { levels } \\
\text { TH-immunohistochemistry }\end{array}$ & $\begin{array}{l}\text { Test affective } \\
\text { disorders }\end{array}$ & 111 \\
\hline & $\begin{array}{l}\text { Unilateral 6-OHDA- } \\
\text { lesioned rat }\end{array}$ & $\begin{array}{l}\text { Body AIMs score } \\
\text { Contralateral } \\
\text { rotational } \\
\text { behavior }\end{array}$ & $\begin{array}{l}\Delta \text { FosB expression } \\
\text { ERKI/2 phosphorylation }\end{array}$ & $\begin{array}{l}\text { Test L-DOPA- } \\
\text { induced dyskinesia }\end{array}$ & 117 \\
\hline $\begin{array}{l}\text { Curcumin, naringenin } \\
\text { (green tea) }\end{array}$ & $\begin{array}{l}\text { Unilateral 6-OHDA- } \\
\text { lesioned rat }\end{array}$ & None & $\begin{array}{l}\text { TH-positive cells } \\
\text { Dopamine levels }\end{array}$ & $\begin{array}{l}\text { Test symptomatic } \\
\text { therapy }\end{array}$ & 94 \\
\hline $\begin{array}{l}\text { Epigallocatechin } \\
\text { derivatives (green tea) }\end{array}$ & $\begin{array}{l}\text { Unilateral 6-OHDA- } \\
\text { lesioned rat }\end{array}$ & None & $\begin{array}{l}\text { ROS and NO levels } \\
\text { Lipid peroxidation } \\
\text { NO synthase }\end{array}$ & $\begin{array}{l}\text { Test symptomatic } \\
\text { therapy }\end{array}$ & 95 \\
\hline $\begin{array}{l}\text { Bilobalide (Ginkgo } \\
\text { biloba) }\end{array}$ & $\begin{array}{l}\text { Unilateral 6-OHDA- } \\
\text { lesioned rat }\end{array}$ & $\begin{array}{l}\text { Locomotor activity } \\
\text { Rotational } \\
\text { behavior }\end{array}$ & $\begin{array}{l}\text { TH-positive cells } \\
\text { NF-אB p65 expression }\end{array}$ & $\begin{array}{l}\text { Test symptomatic } \\
\text { therapy }\end{array}$ & 96 \\
\hline $\begin{array}{l}\text { G. biloba (acetone- } \\
\text { water extract) }\end{array}$ & $\begin{array}{l}\text { MPTP-lesioned mouse } \\
\text { (C57) }\end{array}$ & None & $\begin{array}{l}\text { Dopamine levels } \\
\text { MAO activity }\end{array}$ & $\begin{array}{l}\text { Test symptomatic } \\
\text { therapy }\end{array}$ & 97 \\
\hline $\begin{array}{l}\text { Kavain (Piper } \\
\text { methysticum) }\end{array}$ & $\begin{array}{l}\text { MPTP-lesioned mouse } \\
\text { (C57BL/6) }\end{array}$ & None & $\begin{array}{l}\text { TH-immunocyto- } \\
\text { chemistry } \\
\text { Dopamine and its } \\
\text { metabolite levels }\end{array}$ & $\begin{array}{l}\text { Test symptomatic } \\
\text { therapy }\end{array}$ & 98 \\
\hline Ginsenoside RgI & $\begin{array}{l}\text { MPTP-lesioned mouse } \\
\text { (C57BL) }\end{array}$ & None & $\begin{array}{l}\text { TH-immunohistochemistry } \\
\mathrm{NO} \text { synthase } \\
\mathrm{Bcl}-2, \mathrm{Bcl}-\mathrm{xl} \text {, and } \mathrm{Bax} \\
\text { levels } \\
\text { Caspase-3 levels }\end{array}$ & $\begin{array}{l}\text { Test symptomatic } \\
\text { therapy }\end{array}$ & 99 \\
\hline $\begin{array}{l}\text { Echinacoside } \\
\text { (Cistanches salsa) }\end{array}$ & $\begin{array}{l}\text { MPTP-lesioned mouse } \\
\text { (C57BL/6) }\end{array}$ & Locomotion test & $\begin{array}{l}\text { TH expression } \\
\text { Dopamine and its } \\
\text { metabolite levels } \\
\text { Caspase- } 3 \text { and }-8 \text { levels }\end{array}$ & $\begin{array}{l}\text { Test symptomatic } \\
\text { therapy }\end{array}$ & 100 \\
\hline $\begin{array}{l}\text { Celastrol (Celastraceae } \\
\text { family) }\end{array}$ & $\begin{array}{l}\text { MPTP-lesioned mouse } \\
\text { 3-Nitropropionic } \\
\text { acid-lesioned rat }\end{array}$ & None & $\begin{array}{l}\text { Dopaminergic neurons } \\
\text { Dopamine depletion } \\
\text { Heat shock protein } \\
\text { levels } \\
\text { TNF- } \alpha \text { and NF-אB } \\
\text { expression }\end{array}$ & $\begin{array}{l}\text { Test symptomatic } \\
\text { therapy }\end{array}$ & 101 \\
\hline $\begin{array}{l}\text { Paeoniflorin } \\
\text { (Paeoniae alba) }\end{array}$ & $\begin{array}{l}\text { MPTP-lesioned mouse } \\
\text { (C57BL/6) }\end{array}$ & Pole test & $\begin{array}{l}\text { TH-positive cells } \\
\text { Microglial and astrocytic } \\
\text { activation } \\
\text { Adenosine } \mathrm{A}_{\text {, receptor }} \\
\text { activation } \\
\text { MPP }^{+} \text {assay }\end{array}$ & $\begin{array}{l}\text { Test symptomatic } \\
\text { therapy } \\
\text { Test neuro- } \\
\text { inflammation }\end{array}$ & 102 \\
\hline
\end{tabular}


Table I (Continued)

\begin{tabular}{|c|c|c|c|c|c|}
\hline $\begin{array}{l}\text { Bioactive } \\
\text { component/herbal } \\
\text { medicine }\end{array}$ & Toxic model & $\begin{array}{l}\text { Behavioral } \\
\text { function }\end{array}$ & Biochemical analysis & $\begin{array}{l}\text { Application for } \\
\text { the model }\end{array}$ & Reference \\
\hline \multirow[t]{2}{*}{ Resveratrol } & $\begin{array}{l}\text { MPTP-lesioned mouse } \\
\text { (Balb/c) }\end{array}$ & $\begin{array}{l}\text { Motor } \\
\text { coordination } \\
\text { impairment }\end{array}$ & Hydroxy radical levels & $\begin{array}{l}\text { Test symptomatic } \\
\text { therapy }\end{array}$ & 103 \\
\hline & $\begin{array}{l}\text { MPTP-lesioned mouse } \\
\text { (C57BL/6) }\end{array}$ & None & $\begin{array}{l}\text { Dopamine depletion } \\
\text { TH-immunopositive } \\
\text { cells }\end{array}$ & $\begin{array}{l}\text { Test symptomatic } \\
\text { therapy }\end{array}$ & 104 \\
\hline $\begin{array}{l}\text { Baicalein (Scutellaria } \\
\text { baicalensis) }\end{array}$ & $\begin{array}{l}\text { MPTP-lesioned mouse } \\
\text { (C57BL/6) }\end{array}$ & $\begin{array}{l}\text { Spontaneous } \\
\text { motor activity } \\
\text { Pole test }\end{array}$ & $\begin{array}{l}\text { Dopamine and serotonin } \\
\text { levels }\end{array}$ & $\begin{array}{l}\text { Test symptomatic } \\
\text { therapy }\end{array}$ & 105 \\
\hline \multirow[t]{2}{*}{$\begin{array}{l}\text { Acanthopanax senticosus } \\
\text { (ethanol extract) }\end{array}$} & MPTP-lesioned rat & Pole test & TH-positive cells & $\begin{array}{l}\text { Test symptomatic } \\
\text { therapy }\end{array}$ & 110 \\
\hline & & $\begin{array}{l}\text { Catalepsy test } \\
\text { (bradykinesia } \\
\text { and depressive } \\
\text { behavior) }\end{array}$ & & Test PD catalepsy & \\
\hline Sesamin (A. senticosus) & Rotenone-lesioned rat & $\begin{array}{l}\text { Pole test } \\
\text { Catalepsy test }\end{array}$ & $\begin{array}{l}\text { TH-positive cells } \\
\text { GDNF-positive cells }\end{array}$ & $\begin{array}{l}\text { Test symptomatic } \\
\text { therapy }\end{array}$ & 107 \\
\hline \multirow[t]{2}{*}{$\begin{array}{l}\text { Withania somnifera } \\
\text { (root powder) }\end{array}$} & $\begin{array}{l}\text { MPTP-lesioned mouse } \\
\text { (Albino) }\end{array}$ & Rotarod test & TBARS levels & $\begin{array}{l}\text { Test symptomatic } \\
\text { therapy }\end{array}$ & 108 \\
\hline & & $\begin{array}{l}\text { Hang test } \\
\text { Stride length } \\
\text { measurement }\end{array}$ & $\begin{array}{l}\text { SOD and catalase } \\
\text { activities }\end{array}$ & $\begin{array}{l}\text { Test chronic } \\
\text { stress-induced } \\
\text { pathology }\end{array}$ & \\
\hline $\begin{array}{l}\text { Purslane (Portulaca } \\
\text { oleracea) }\end{array}$ & Rotenone-lesioned rat & None & $\begin{array}{l}\text { Striatal calcium levels } \\
\text { Dopamine metabolite } \\
\text { levels } \\
\text { Complex I activity }\end{array}$ & $\begin{array}{l}\text { Test symptomatic } \\
\text { therapy }\end{array}$ & 109 \\
\hline Amantadine & $\begin{array}{l}\text { PD patients } \\
\text { (clinical trials) }\end{array}$ & $\begin{array}{l}\text { Total dyskinesia } \\
\text { score }\end{array}$ & None & $\begin{array}{l}\text { Test L-DOPA- } \\
\text { induced dyskinesia }\end{array}$ & 112 \\
\hline $\begin{array}{l}\text { Nabilone (Cannabinoid } \\
\text { receptor agonist) }\end{array}$ & $\begin{array}{l}\text { PD patients } \\
\text { (clinical trials) }\end{array}$ & $\begin{array}{l}\text { Total dyskinesia } \\
\text { score }\end{array}$ & $\begin{array}{l}\text { GABA reuptake } \\
\text { GABA transmission }\end{array}$ & $\begin{array}{l}\text { Test L-DOPA- } \\
\text { induced dyskinesia }\end{array}$ & 113 \\
\hline Aripiprazole & $\begin{array}{l}\text { PD patients } \\
\text { (clinical trials) }\end{array}$ & $\begin{array}{l}\text { Trunk } \\
\text { measurement } \\
\text { (AIMs score) }\end{array}$ & None & $\begin{array}{l}\text { Test L-DOPA- } \\
\text { induced dyskinesia }\end{array}$ & 114 \\
\hline \multirow[t]{2}{*}{ Endocannabinoids } & $\begin{array}{l}\text { MPTP-induced } \\
\text { cynomolgus monkey }\end{array}$ & $\begin{array}{l}\text { Total dyskinesia } \\
\text { score (movement } \\
\text { range, posture, } \\
\text { bradykinesia) }\end{array}$ & None & $\begin{array}{l}\text { Test PD } \\
\text { generation }\end{array}$ & 115 \\
\hline & & $\begin{array}{l}\text { Locomotor and } \\
\text { motor function } \\
\text { tests }\end{array}$ & & $\begin{array}{l}\text { Test L-DOPA- } \\
\text { induced dyskinesia }\end{array}$ & \\
\hline Stepholidine & 6-OHDA-lesioned rat & $\begin{array}{l}\text { AIMs score } \\
\text { Locomotive } \\
\text { score } \\
\text { Contralateral } \\
\text { rotational } \\
\text { behavior }\end{array}$ & $5-\mathrm{HT}_{\text {IA }}$ mRNA levels & $\begin{array}{l}\text { Test L-DOPA- } \\
\text { induced dyskinesia }\end{array}$ & 116 \\
\hline Lycopene & Rotenone-lesioned rat & Cognitive decline & $\begin{array}{l}\text { Glutathione levels } \\
\text { SOD and catalase } \\
\text { activities } \\
\text { NADH and AChE } \\
\text { activities }\end{array}$ & $\begin{array}{l}\text { Test PD-induced } \\
\text { cognitive } \\
\text { disorders }\end{array}$ & 118 \\
\hline
\end{tabular}

Abbreviations: 5-HT, 5-hydroxytryptamine; 6-OHDA, 6-hydroxydopamine; AChE, acetylcholinesterase; AIMs, abnormal involuntary movements; GABA, $\gamma$-aminobutyric acid; GDNF, glial cell line-derived neurotrophic factor; ERK, extracellular signal-regulated kinases; L-DOPA, L-3,4-dihydroxyphenylalanine; MAO, monoamine oxidase; $\mathrm{MPP}^{+}$, I-methyl-4-phenylpyridinium; MPTP, I-methyl-4-phenyl-1,2,3,6-tetrahydropyridine; mRNA, messenger RNA; NF- $\mathrm{KB}$, nuclear transcription factor- $\mathrm{KB}$; NADH, reduced nicotinamide adenine dinucleotide; NO, nitric oxide; PD, Parkinson's disease; ROS, reactive oxygen species; SOD, superoxide dismutase; TBARS, thiobarbituric acid-reactive substance; TH, tyrosine hydroxylase; TNF- $\alpha$, tumor necrosis factor- $\alpha$. 
induced dyskinesia in the 6-OHDA-induced rat model of PD. ${ }^{116}$ GPS and the ethanol extract from G. pentaphyllum also attenuate the development of L-DOPA-induced dyskinesia by modulating the biomarker activities of delta-FosB expression and extracellular signal-regulated kinases (ERK1/2) phosphorylation in the 6-OHDA-induced rat model of PD. ${ }^{117}$

\section{Effects on L-DOPA-induced memory dysfunctions or cognitive disorders}

Lycopene exerts protective effects on the cognitive decline that is observed in the rotenone-induced model of PD. ${ }^{118}$ Recently, it has been demonstrated that (-)-sesamin from Asiasarum heterotropoides F. Maekawa var. mandshuricum, and GPS and the ethanol extract from G. pentaphyllum have neuroprotective effects on the L-DOPA-induced memory dysfunctions by modulating phosphorylation of ERK1/2, cyclic AMP-response element binding protein, and NMDA receptor in the hippocampus of MPTP-induced mouse model of PD (Zhao et al, unpublished data, 2016; Kim et al, unpublished data, 2016).

\section{Conclusion}

Rats and mice are widely used as models of PD. Nonhuman primates are also used for the PD models. Neurotoxin models are animal models of end-stage $\mathrm{PD}$, although researchers are working toward developing a progressive toxic model. In contrast, genetic models are created through overexpression or knockout methodology and occasionally through knockin or conditional methodology. However, neither neurotoxin nor genetic animal models display all of the pathophysiological symptoms of PD. PD is a multi-symptomatic disease that mainly arises through environmental neurodegenerative factors and genetic condition. Because a single model can have advantages and disadvantages, further studies might combine genetic models with neurotoxin models and/or double neurotoxin models by each PD-related research plan.

In addition, the effects of various bioactive components from natural herbal products have been reported. However, the clinical applications of these components as therapeutic drugs for PD remain undeveloped: these reasons are raised as the bioactive components are not able to show the desirable drug efficacy because of the recurrent limitation with PD animal models and may also induce the neurotoxicity by their long-term administrations in the animal models.

\section{Acknowledgment}

This research was financially supported by the National Research Foundation of Korea (2013-R1A1A2058230) (2015), Republic of Korea.

\section{Disclosure}

The authors report no conflicts of interest in this work.

\section{References}

1. Fearnley JM, Lees AJ. Ageing and Parkinson's disease: substantia nigra regional selectivity. Brain. 1991;114:2283-2301.

2. Marsden CD. Parkinson's disease. J Neurol Neurosurg Psychiatry. 1994;57:672-681.

3. Fahn S. Description of Parkinson's disease as a clinical syndrome. Ann NY Acad Sci. 2003;991:1-14.

4. Yacoubian TA, Standaert DG. Targets for neuroprotection in Parkinson's disease. Biochim Biophys Acta. 2009;1792:676-687.

5. Jankovic J. Motor fluctuations and dyskinesias in Parkinson's disease: clinical manifestations. Mov Disord. 2005;20:S11-S16.

6. Olanow CW, Damier P, Goetz CG, et al. Multicenter, open-label, trial of sarizotan in Parkinson disease patients with levodopa-induced dyskinesias (the Splendid Study). Clin Neuropharmacol. 2004;27:58-62.

7. Cummings JL. Depression and Parkinson's disease: a review. Am J Psychiatry. 1992;149:443-454.

8. Walsh K, Bennett G. Parkinson's disease and anxiety. Postgrad Med J. 2001;77:89-93.

9. Maricle RA, Nutt JG, Valentine RJ, et al. Dose-response relationship of levodopa with mood and anxiety in fluctuating Parkinson's disease: a double-blind, placebo-controlled study. Neurology. 1995; 45:1757-1760.

10. Funkiewiez A, Ardouin C, Cools R, et al. Effects of levodopa and subthalamic nucleus stimulation on cognitive and affective functioning in Parkinson's disease. Mov Disord. 2006;21:1656-1662.

11. Decamp E, Schneider JS. Interaction between nicotinic and dopaminergic therapies on cognition in a chronic Parkinson model. Brain Res. 2009;1262:109-114.

12. Jackson-Lewis V, Blesa J, Przedborski S. Animal models of Parkinson's disease. Parkinsonism Relat Disord. 2012;18 (Suppl 1):S183-S185.

13. Xu Y, Deng YL, Qing H. The phosphorylation of $\alpha$-synuclein: development and implication for the mechanism and therapy of the Parkinson's disease. J Neurochem. 2015;135:4-18.

14. Tufekci KU, Genc S, Genc K. The endotoxin-induced neuroinflammation model of Parkinson's disease. Parkinsons Dis. 2011;2011:487450.

15. Dawson TM, Ko HS, Dawson VL. Genetic animal models of Parkinson's disease. Neuron. 2010;66:646-661.

16. Cohen G. Oxy-radical toxicity in catecholamine neurons. Neurotoxicology. 1984;5:77-82.

17. Soto-Otero R, Méndez-Álvarez E, Hermida-Ameijeiras Á, et al. Autoxidation and neurotoxicity of 6-hydroxydopamine in the presence of some antioxidants: potential implication in relation to the pathogenesis of Parkinson's disease. J Neurochem. 2000;74:1605-1612.

18. Blandini F, Armentero MT, Martignoni E. The 6-hydroxydopamine model: news from the past. Parkinsonism Relat Disord. 2008;14 (Suppl 2):S124-S129.

19. Alves da Costa C, Dunys J, Brau F, et al. 6-Hydroxydopamine but not 1-methyl-4-phenylpyridinium abolishes $\alpha$-synuclein anti-apoptotic phenotype by inhibiting its proteasomal degradation and by promoting its aggregation. J Biol Chem. 2006;281:9824-9831.

20. Crowther RA, Daniel SE, Goedert M. Characterisation of isolated $\alpha$-synuclein filaments from substantia nigra of Parkinson's disease brain. Neurosci Lett. 2000;292:128-130.

21. Ungerstedt U. 6-Hydroxy-dopamine induced degeneration of central monoamine neurons. Eur J Pharmacol. 2006;281:9824-9831.

22. Schwarting RKW, Huston JP. The unilateral 6-hydroxydopamine lesion model in behavioral brain research. Analysis of functional deficits, recovery and treatments. Prog Neurobiol. 1996;49:215-266.

23. Curtius HC, Wolfensberger M, Steinmann B, et al. Mass fragmentography of dopamine and 6-hydroxydopamine: application to the determination of dopamine in human brain biopsies from the caudate nucleus. J Chromatogr A. 1974;99:529-540. 
24. Andrew R, Watson DG, Best SA, et al. The determination of hydroxydopamines and other trace amines in the urine of parkinsonian patients and normal controls. Neurochem Res. 1993;18:1175-1177.

25. Terzioglu M, Galter D. Parkinson's disease: genetic versus toxin-induced rodent models. FEBS J. 2008;275:1384-1391.

26. Bové J, Perier C. Neurotoxin-based models of Parkinson's disease. Neuroscience. 2012;211:51-76.

27. Heikkila RE, Hess A, Duvoisin RC. Dopaminergic neurotoxicity of 1-methyl-4-phenyl-1,2,5,6-tetrahydropyridine in mice. Science. 1984;224:1451-1453.

28. Nicklas WJ, Vyas I, Heikkila RE. Inhibition of NADH-linked oxidation in brain mitochondria by 1-methyl-4-phenyl-pyridine, a metabolite of the neurotoxin, 1-methyl-4-phenyl-1,2,5,6-tetrahydropyridine. Life Sci. 1985;36:2503-2508.

29. McCormack AL, Mak SK, Shenasa M, et al. Pathological modifications of $\alpha$-synuclein in 1-methyl-4-phenyl-1,2,3,6-tetrahydropyridine (MPTP)-treated squirrel monkeys. Neuropathol Exp Neurol. 2008; 67:793-802.

30. Purisai MG, McCormack AL, Langston WJ, et al. $\alpha$-Synuclein expression in the substantia nigra of MPTP-lesioned non-human primates. Neurobiol Dis. 2005;20:898-906.

31. Collier TJ, Kanaan NM, Kordower JH. Aging as a primary risk factor for Parkinson's disease: evidence from studies of non-human primates. Nat Rev Neurosci. 2011;12:359-366.

32. Eriksen N, Stark AK, Pakkenberg B. Age and Parkinson's disease-related neuronal death in the substantia nigra pars compacta. J Neural Transm Suppl. 2009;73:203-213.

33. McCormack AL, Mak SK, Henderson JM, et al. $\alpha$-Synuclein suppression by targeted small interfering RNA in the primate substantia nigra. PLoS One. 2010;5:e12122.

34. Agid Y, Javoy-Agid F, Ruberg M. Biochemistry of neurotransmitter in Parkinson's disease. In: Marsden CD, Fahn S, editors. Movement Disorders, Vol 2. London: Butterworths; 1987:166-230.

35. Langston JW, Forno LS, Tetrud J, et al. Evidence of active nerve cell degeneration in the substantia nigra of human years after 1-methyl-4-phenyl-1,2,3,6-tetrahydropyridine exposure. Ann Neurol. 1999;46:598-605.

36. Dhillon AS, Tarbutton GL, Levin JL, et al. Pesticide/environmental exposures and Parkinson's disease in East Texas. J Agromed. 2008;13: 37-48.

37. Greenamyre JT, Cannon JR, Drolet R, Mastroberardino PG. Lessons from the rotenone model of Parkinson's disease. Trends Pharmacol Sci. 2010;31:141-142.

38. Wu YN, Johnson SW. Dopamine oxidation facilitates rotenonedependent potentiation of N-methyl-D-aspartate currents in rat substantia nigra dopamine neurons. Neuroscience. 2011;195:138-144.

39. Berry C, Vecchia CL, Nicotera P. Paraquat and Parkinson's disease. Cell Death Differ. 2010;17:1115-1125.

40. Miller GW. Paraquat: the red herring of Parkinson's disease research. Toxicol Sci. 2007;100:1-2.

41. Brooks AI, Chadwick CA, Gelbard HA, et al. Paraquat elicited neurobehavioral syndrome caused by dopaminergic neuron loss. Brain Res. 1999;823:1-10.

42. Prasad K, Tarasewicz E, Mathew J, et al. Toxicokinetics and toxicodynamics of paraquat accumulation in mouse brain. Exp Neurol. 2009;215:358-367.

43. Manning-Bog AB, McCormack AL, Li J, et al. The herbicide paraquat causes up-regulation and aggregation of $\alpha$-synuclein in mice. Paraquat and $\alpha$-synuclein. J Biol Chem. 2002;277:1641-1644.

44. Trulson ME, Cannon MS, Faegg TS, Raese JD. Effects of chronic methamphetamine on the nigral-striatal dopamine system in rat brain: tyrosine hydroxylase immunochemistry and quantitative light microscopic studies. Brain Res Bull. 1985;15:569-577.

45. Cashaw JL. Tetrahydropapaveroline in brain regions of rats after acute ethanol administration. Alcohol. 1993;10:133-138.

46. Nagatsu T. Isoquinoline neurotoxin in the brain and Parkinson's disease. Neurosci Res. 1997;29:99-111.
47. Storch A, Ott S, Hwang YI, et al. Selective dopaminergic neurotoxicity of isoquinoline derivatives related to Parkinson's disease: studies using heterologous expression systems of the dopamine transporter. Biochem Pharmacol. 2002;63:909-920.

48. Barbeau A. Manganese and extrapyramidal disorders. Neurotoxicology. 1984;5:13-35.

49. Corti O, Lesage S, Brice A. What genetics tells us about the causes and mechanisms of Parkinson's disease. Physiol Rev. 2011;91: 1161-1218.

50. Polymeropoulos MH, Lavedan C, Leroy E, et al. Mutation in the alphasynuclein gene identified in families with Parkinson's disease. Science. 1997;276:2045-2047.

51. Lees AJ, Hardy J, Revesz T. Parkinson's disease. Lancet. 2009;373: 2055-2066.

52. Singleton AB. Altered alpha-synuclein homeostasis causing Parkinson's disease: the potential roles of dardarin. Trends Neurosci. 2005;28:416-421.

53. Fornai F, Schlüter OM, Lenzi P, et al. Parkinson-like syndrome induced by continuous MPTP infusion: convergent roles of the ubiquitin proteasome system and $\alpha$-synuclein. Proc Nat Acad Sci U S A. 2005;102:3413-3418.

54. Cannon JR, Tapias VM, Na HM, et al. A highly reproducible rotenone model of Parkinson's disease. Neurobiol Dis. 2009;34:279-290.

55. Abeliovich A, Schmitz Y, Fariñas I, et al. Mice lacking alpha-synuclein display functional deficits in the nigrostriatal dopamine system. Neuron. 2000;25:239-252.

56. Thiruchelvam MJ, Powers JM, Cory-Slechta DA, Richfield RK. Risk factors for dopaminergic neuron loss in human $\alpha$-synuclein transgenic mice. Eur J Neurosci. 2004;19:845-854.

57. Nuber S, Petrasch-Parwez, Winner B, et al. Neurodegeration and motor dysfunction in a conditional model of Parkinson's disease. J Neurosci. 2008;28:2471-2484.

58. Iwatsubo T. Pathological biochemistry of alpha-synucleinopathy. Neuropathology. 2007:27;474-478.

59. Foulds PG, Mitchell JD, Parker A, et al. Phosphorylated alpha-synuclein can be detected in blood plasma and is potentially a useful biomarker for Parkinson's disease. FASEB J. 2011;25:4127-4137.

60. Wang Y, Shi M, Chung KA, et al. Phosphorylated alpha-synuclein in Parkinson's disease. Sci Transl Med. 2012;4:121ra20.

61. Biskup S, Moore DJ, Celsi F, et al. Localization of LRRK2 to membranous and vesicular structures in mammalian brain. Ann Neurol. 2006;60:557-569.

62. Zimprich A, Biskup S, Leitner P, Lichtner P. Mutations in LRRK2 cause autosomal-dominant parkinsonism with pleomorphic pathology. Neuron. 2001;44:601-607.

63. Greggio E, Jain S, Kingsbury A, et al. Kinase activity is required for the toxic effects of mutant LRRK2/dardarin. Neurobiol Dis. 2006;23: 329-341.

64. Albrecht M. LRRK2 mutations and Parkinsonism. Lancet. 2005;365: $12-30$.

65. Ross OA, Toft M, Whittle AJ, et al. Lrrk2 and Lewy body disease. Ann Neurol. 2006;59:388-393.

66. Smith WW, Pei Z, Jiang H, et al. Kinase activity of mutant LRRK2 mediates neuronal toxicity. Nat Neurosci. 2006;9:1231-1233.

67. Wang D, Tang B, Zhao G, et al. Dispensable role of Drosophila ortholog of LRRK2 kinase activity in survival of dopaminergic neurons. Mol Neurodegener. 2008;3:3.

68. Ramirez A, Heimbach A, Gründemann J, et al. Hereditary parkinsonism with dementia is caused by mutations in ATP13A2, encoding a lysosomal type 5 P-type ATPase. Nat Genet. 2006;38:1184-1191.

69. Lücking CB, Dürr A, Bonifati V, et al. Association between early-onset Parkinson's disease and mutations in the Parkin Gene. $N$ Engl J Med. 2000;342:1560-1567.

70. Gasser T. Molecular pathogenesis of Parkinson disease: insights from genetic studies. Expert Rev Mol Med. 2009;11:e22.

71. Heutink P. PINK-1 and DJ-1 - new genes for autosomal recessive Parkinson's disease. J Neural Transm Suppl. 2006;70:215-219. 
72. Shimura H, Hattori N, Kubo S, et al. Familial Parkinson disease gene product, parkin, is a ubiquitin-protein ligase. Nat Genet. 2000;25:302-305.

73. Moore DJ, Dawson TM. Value of genetic models in understanding the cause and mechanisms of Parkinson's disease. Curr Neurol Neurosci. 2008;8:288-296.

74. Shin JH, Ko HS, Kang H, et al. PARIS (ZNF746) repression of PGC-1 $\alpha$ contributes to neurodegeneration in Parkinson's disease. Cell. 2011;144:689-702.

75. Silvestri L, Caputo V, Bellacchio E, et al. Mitochondrial import and enzymatic activity of PINK1 mutants associated to recessive parkinsonism. Hum Mol Genet. 2005;14:3477-3492.

76. Gandhi S, Wood-Kaczmar A, Yao Z, et al. PINK1-associated Parkinson's disease is caused by neuronal vulnerability to calcium-induced cell death. Mol Cell. 2009;33:627-638.

77. Gautier CA, Kitada T, Shen J, et al. Loss of PINK1 causes mitochondrial functional defects and increased sensitivity to oxidative stress. Proc Nat Acad Sci U S A. 2008;105:11364-11369.

78. Deng H, Dodson MW, Huang H, Guo M. The Parkinson's disease genes pink1 and parkin promote mitochondrial fission and/or inhibit fusion in Drosophila. Proc Nat Acad Sci U S A. 2008;105:14503-14508.

79. Narendra DP, Jin SM, Tanaka A, et al. PINK1 is selectively stabilized on impaired mitochondria to activate parkin. PLoS Biol. 2010;8:e1000298.

80. Zhang L, Shimoji M, Thomas B, et al. Mitochondrial localization of the Parkinson's disease related protein DJ-1: implications for pathogenesis. Hum Mol Genet. 2005;14:2063-2073.

81. Andres-Mateos E, Perier C, Zhang L, et al. DJ-1 gene deletion reveals that DJ-1 is an atypical peroxiredoxin-like peroxidase. Proc Nat Acad Sci U S A. 2007;104:14807-14812.

82. Bonifati V, Rizzu P, van Baren MJ, et al. Mutations in the $D J-1$ gene associated with autosomal recessive early-onset parkinsonism. Science. 2003;299:256-259.

83. Dehay B, Ramirez A, Martinez-Vicente M, et al. Loss of P-type ATPase ATP13A2/PARK9 function induces general lysosomal deficiency and leads to Parkinson disease neurodegeneration. Proc Natl Acad Sci USA. 2012;9:9611-9616.

84. Usenovic M, Tresse E, Mazzulli JR, et al. Deficiency of ATP13A2 leads to lysosomal dysfunction, $\alpha$-synuclein accumulation, and neurotoxicity. $J$ Neurosci. 2012;32:4240-4246.

85. Hefti F, Melamed E, Wurtman RJ. Partial lesions of the dopaminergic nigrostriatal system in rat brain: biochemical characterization. Brain Res. 1980;195:123-137.

86. Sheikh N, Ahm A, Siripurapu KB, et al. Effect of Bacopa monniera on stress induced changes in plasma corticosterone and brain monoamines in rats. J Ethnopharmacol. 2007;111:671-676.

87. Newcomer JW, Krystal JH. NMDA receptor regulation of memory and behavior in humans. Hippocampus. 2001;11:529-542.

88. Buter TC, van den Hout A, Matthews FE, et al. Dementia and survival in Parkinson disease: a 12-year population study. Neurology. 2008;70:1017-1022.

89. Visanji NP, O’Neill MJ, Duty S. Nicotine, but neither the alpha4beta2 ligand RJR2403 nor an alpha7 nAChR subtype selective agonist, protects against a partial 6-hydroxydopamine lesion of the rat median forebrain bundle. Neuropharmacology. 2006;51:506-516.

90. Szeto HH. Development of mitochondria-targeted aromatic-cationic peptides for neurodegenerative diseases. Ann N Y Acad Sci. 2008; 1147:112-121.

91. Ho YJ, Ho SC, Pawlak CR, Yeh KY. Effects of d-cycloserine on MPTPinduced behavioral and neurological changes: potential for treatment of Parkinson's disease dementia. Behav Brain Res. 2011;219:280-290.

92. Hussain G, Manyam BV. Mucuna pruriens proves more effective than L-DOPA in Parkinson's disease animal model. Phytother Res. 1997;11:419-423.

93. Choi HS, Park MS, Kim SH, et al. Neuroprotective effects of herbal ethanol extracts from Gynostemma pentaphyllum in the 6-hydroxydopamine-lesioned rat model of Parkinson's disease. Molecules. 2010;15:2814-2824.
94. Zbarsky V, Datla KP, Parkar S, et al. Neuroprotective properties of the natural phenolic antioxidants curcumin and naringenin but not quercetin and fisetin in a 6-OHDA model of Parkinson's disease. Free Radic Res. 2005;39:1119-1125.

95. Guo SH, Yan JQ, Yang TB, et al. Protective effects of green tea polyphenols in the 6-OHDA rat model of Parkinson's disease through inhibition of ROS-NO pathway. Biol Psychiatry. 2007;62:1353-1362.

96. Li LY, Zhao XL, Fei XF, et al. Bilobalide inhibits 6-OHDA-induced activation of NF-kB and loss of dopaminergic neurons in rat substantia nigra. Acta Pharmacol Sin. 2008;29:539-547.

97. Wu WR, Zhu XZ. Involvement of monoamine oxidase inhibition in neuroprotective and neurorestorative effects of Ginkgo biloba extract against MPTP-induced nigrostriatal dopaminergic toxicity in C57 mice. Life Sci. 1999;65:157-164

98. Schmidt N, Ferger B. Neuroprotective effects of $( \pm)$-Kavain in the MPTP mouse model of Parkinson's disease. Synapse. 2001;40:47-54.

99. Chen XC, Chen Y, Zhu YG, et al. Protective effect of ginsenoside Rg1 against MPTP-induced apoptosis in mouse substantia nigra neurons Acta Pharmacol Sin. 2002;23:829-834.

100. Geng XC, Tian XF, Tu PF, Pu XP. Neuroprotective effects of echinacoside in the mouse MPTP model of Parkinson's disease. Eur $J$ Pharmacol. 2007;564:66-74.

101. Cleren C, Calingasan NY, Chen J, Beal MF. Celastrol protects against MPTP- and 3-nitropropionic acid-induced neurotoxicity. J Neurochem. 2005;94:995-1004.

102. Liu HQ, Zhang WY, Luo XT, Ye Y, Zhu XZ. Paeoniflorin attenuates neuroinflammation and dopaminergic neurodegeneration in the MPTP model of Parkinson's disease by activation of adenosine A1 receptor. Br J Pharmacol. 2006;148:314-325.

103. Lu KT, Ko MC, Chen BY, et al. Neuroprotective effects of resveratrol on MPTP-induced neuron loss mediated by free radical scavenging. J Agric Food Chem. 2008;56:6910-6913.

104. Blanchet J, Longpré F, Bureau G, et al. Resveratrol, a red wine polyphenol, protects dopaminergic neurons in MPTP-treated mice. Prog Neuropsychopharmacol Biol Psychiatry. 2008;32:1243-1250.

105. Cheng YX, He GR, Mu X, et al. Neuroprotective effect of baicalein against MPTP neurotoxicity: behavioral, biochemical and immunohistochemical profile. Neurosci Lett. 2008;441:16-20.

106. Wang P, Niu L, Gao L, et al. Neuroprotective effect of gypenosides against oxidative injury in the substantia nigra of a mouse model of Parkinson's disease. J Int Med Res. 2010;38:1084-1092.

107. Fujikawa T, Miguchi S, Kanada N, et al. Acanthopanax senticosus Harms as a prophylactic for MPTP-induced Parkinson's disease in rats. J Ethnopharmacol. 2005;97:375-381.

108. Sankar SR, Manivasagam T, Krishnamurti A, Ramanathan M. The neuroprotective effect of Withania somnifera root extract in MPTPintoxicated mice: an analysis of behavioral and biochemical variables. Cell Mol Biol Lett. 2007;12:473-481.

109. Moneim AEA. The neuroprotective effects of Purslane (Portulaca oleracea) on rotenone-induced biochemical changes and apoptosis in brain of rat. CNS Neurol Disord Drug Targets. 2013;12:830-841.

110. Fujikawa T, Kanada N, Shimada A, et al. Effect of sesamin in Acanthopanax senticosus HARMS on behavioral dysfunction in rotenoneinduced parkinsonian rats. Biol Pharm Bull. 2005;28:169-172.

111. Shin KS, Zhao TT, Choi HS, et al. Effects of gypenosides on anxiety disorders in MPTP-lesioned mouse model of Parkinson's disease. Brain Res. 2014;1567:57-65.

112. Snow BJ, Macdonald L, Mcauley D, Wallis W. The effect of amantadine on levodopa-induced dyskinesias in Parkinson's disease: a double-blind, placebo-controlled study. Clin Neuropharmacol. 2000;23:82-85.

113. Sieradzan KA, Fox SH, Hill M, et al. Cannabinoids reduce levodopainduced dyskinesia in Parkinson's disease: a pilot study. Neurology. 2001;5:2108-2111

114. Meco G, Stirpe P, Edito F, et al. Aripiprazole in L-dopa-induced dyskinesias: a one-year open-label pilot study. J Neural Transm. 2009; 116:881-884. 
115. van der Stelt M, Fox SH, Hill M, et al. A role for endocannabinoids in the generation of parkinsonism and levodopa-induced dyskinesia in MPTP-lesioned non-human primate models of Parkinson's disease. FASEB J. 2005;19:1140-1142.

116. Mo J, Zhang H, Yu LP, et al. L-Stepholidine reduced L-DOPA-induced dyskinesia in 6-OHDA-lesioned rat model of Parkinson's disease. Neurobiol Aging. 2010;31:926-936.
117. Shin KS, Zhao TT, Park KH, et al. Gypenosides attenuate the development of L-DOPA-induced dyskinesia in 6-hydroxydopamine-lesioned rat model of Parkinson's disease. BMC Neurosci. 2015;16:23.

118. Kaur H, Chauhan S, Sandhir R. Protective effect of lycopene on oxidative stress and cognitive decline in rotenone induced model of Parkinson's disease. Neurochem Res. 2011;36:1435-1443.

\section{Publish your work in this journal}

Journal of Parkinsonism and Restless Legs Syndrome is an online, open access, peer-reviewed journal. The journal publishes review articles, historical reviews, original research articles, case reports, letters to the editor, clinical teaching cases, neuroradiology highlights, neuropathology highlights, neuropsychiatry highlights, autobiographies, conference

\section{Dovepress}

proceedings, abstracts and book reviews. The manuscript management system is completely online and includes a very quick and fair peerreview system, which is all easy to use. Visit http://www.dovepress.com/ testimonials.php to read real quotes from published authors.

Submit your manuscript here: http://www.dovepress.com/journal-of-parkinsonism--restless-legs-syndrome-journal 